\title{
BMJ Open Utility of the FebriDx point-of-care assay in supporting a triage algorithm for medical admissions with possible COVID-19: an observational
}

\section{cohort study}

\author{
Hamish Houston (D) , ${ }^{1}$ Gavin Deas, ${ }^{2}$ Shivam Naik, ${ }^{3}$ Kamal Shah (D) ${ }^{3}$ Shiras Patel, ${ }^{3}$ \\ Maria Greca Dottori, ${ }^{3}$ Michael Tay, ${ }^{3}$ Sarah Ann Filson, ${ }^{1}$ James Biggin-Lamming, ${ }^{4}$ \\ John Ross, ${ }^{5}$ Natalie Vaughan, ${ }^{6}$ Nidhi Vaid, ${ }^{2}$ Guduru Gopal Rao (D) ,,8 Amit K Amin, ${ }^{7}$ \\ Ankur Gupta-Wright (D) , ${ }^{9,10}$ Laurence John ${ }^{1}$
}

To cite: Houston H, Deas G, Naik S, et al. Utility of the FebriDx point-of-care assay in supporting a triage algorithm for medical admissions with possible COVID-19: an observational cohort study. BMJ Open 2021;11:e049179. doi:10.1136/ bmjopen-2021-049179

- Prepublication history and additional supplemental material for this paper are available online. To view these files, please visit the journal online (http://dx.doi.org/10.1136/ bmjopen-2021-049179).

AG-W and LJ contributed equally.

Received 19 January 2021 Accepted 28 June 2021

Check for updates

(c) Author(s) (or their employer(s)) 2021. Re-use permitted under CC BY-NC. No commercial re-use. See rights and permissions. Published by BMJ.

For numbered affiliations see end of article.

Correspondence to Dr Ankur Gupta-Wright; a.gupta-wright@ucl.ac.uk

\section{ABSTRACT}

Objective To evaluate a triage algorithm used to identify and isolate patients with suspected COVID-19 among medical patients needing admission to hospital using simple clinical criteria and the FebriDx assay.

Design Retrospective observational cohort.

Setting Large acute National Health Service hospital in London, UK.

Participants All medical admissions from the emergency department between 10 August 2020 and 4 November 2020 with a valid SARS-CoV-2 RT-PCR result.

Interventions Medical admissions were triaged as likely, possible or unlikely COVID-19 based on clinical criteria. Patients triaged as possible COVID-19 underwent FebriDx lateral flow assay on capillary blood, and those positive for myxovirus resistance protein $A$ (a host response protein) were managed as likely COVID-19.

Primary outcome measures Diagnostic accuracy (sensitivity, specificity and predictive values) of the algorithm and the FebriDx assay using SARS-CoV-2 RT-PCR from nasopharyngeal swabs as the reference standard.

Results $4.0 \%$ (136) of 3443 medical admissions had RT-PCR confirmed COVID-19. Prevalence of COVID-19 was $46 \%(80 / 175)$ in those triaged as likely, $4.1 \%(50 / 1225)$ in possible and $0.3 \%(6 / 2033)$ in unlikely COVID-19. Using a SARS-CoV-2 RT-PCR reference standard, clinical triage had sensitivity of $96 \%(95 \% \mathrm{Cl} 91 \%$ to $98 \%)$ and specificity of $61.5 \%$ (95\% Cl $59.8 \%$ to $63.1 \%)$, while the triage algorithm including FebriDx had sensitivity of $93 \%$ (95\% Cl $87 \%$ to $96 \%)$ and specificity of $86.4 \%$ (95\% Cl $85.2 \%$ to $87.5 \%$ ). While 2033 patients were deemed not to require isolation using clinical criteria alone, the addition of FebriDx to clinical triage allowed a further 826 patients to be released from isolation, reducing the need for isolation rooms by 9.5 per day, $95 \% \mathrm{Cl} 8.9$ to 10.2 . Ten patients missed by the algorithm had mild or asymptomatic COVID-19.

Conclusions A triage algorithm including the FebriDx assay had good sensitivity and was useful to 'rule-out' COVID-19 among medical admissions to hospital.

\section{Strengths and limitations of this study}

- This was a pragmatic study of a large cohort of consecutive medical admissions enabling a real-world evaluation of the utility of the FebriDx point-of-care assay for COVID-19 triage-a novel application.

- The analyses performed, including estimates of the number of isolation rooms saved and time-to-test result, can inform hospital management when assessing the effectiveness of the FebriDx point-ofcare assay for COVID-19 triage in other settings.

- A single SARS-CoV-2 RT-PCR is an imperfect reference standard for COVID-19 which may impact specificity, and multiple PCR assays were used each with their own performance characteristics.

- The performance of the triage algorithm and the FebriDx assay may differ when used in other populations depending on the underlying prevalence of COVID-19 or other respiratory pathogens.

- The clinical triage criteria were adjusted during the study period to reflect evolving national guidance, which may limit the reproducibility of our results.

\section{INTRODUCTION}

The COVID-19 pandemic, caused by SARS-CoV-2, presents unprecedented challenges for infection prevention and control (IPC) within healthcare facilities worldwide. ${ }^{1}$ Transmission may occur via respiratory droplets, fomites or via airborne routes (following aerosol-generating procedures). ${ }^{2-4}$ Prolonged indoor contact increases transmission, and nosocomial transmission is common. ${ }^{5}{ }^{6}$ Respiratory isolation capacity (neutral or negative pressure side-rooms) is easily saturated within healthcare facilities. ${ }^{1}$ Decisions to isolate patients in need of admission with suspected or possible COVID-19 
must be rapid and accurate to maintain patient flow from emergency departments (EDs), yet minimise risk of nosocomial transmission.

As COVID-19 can present with non-specific symptoms, diagnostic confirmation is often sought by detection of SARS-CoV-2 RNA by reverse transcription-PCR (RT-PCR) from nasopharyngeal swab (NPS). ${ }^{7}$ However, decisions about patient isolation from the ED are usually required before the results of RT-PCR assays are available. ${ }^{89}$ Even near-patient, rapid RT-PCR platforms with assay run times of 1-2hours can be quickly overwhelmed, especially during peaks of COVID-19 incidence. ${ }^{10}{ }^{11}$ Multivariable diagnostic risk models, including clinical criteria and thoracic imaging, are not sufficient, but may be useful as a triage test to ration expensive or scarce point-of-care assays. $^{1213}$

FebriDx (Lumos Diagnostics, Sarasota, Florida, USA) is a lateral flow assay that detects two host response proteins, myxovirus resistance protein A (MxA, positive if $>40 \mathrm{ng} /$ $\mathrm{mL}$ ) and $\mathrm{C}$ reactive protein (CRP, positive if $>20 \mathrm{mg} / \mathrm{L}$ ) in capillary blood samples. MxA is an interferon-induced antiviral host response protein that has been studied as a biomarker to differentiate bacterial and viral respiratory infections. ${ }^{14-17}$ More recently, FebriDx has demonstrated a sensitivity of $93 \%$ and specificity of $86 \%$ for detecting COVID-19 compared with RT-PCR in hospital inpatients including patients with clinically likely COVID-19 and those without symptoms of COVID-19. ${ }^{18}$ FebriDx could be useful as an early triage tool to identify patients with COVID-19 and help guide isolation and IPC in patients needing admission to hospital. ${ }^{18-21}$ We therefore developed and implemented a COVID-19 triage algorithm, supported by FebriDx, to inform patient flow from the ED while awaiting RT-PCR results. Here, we describe the diagnostic performance of this algorithm compared with SARS-CoV-2 RT-PCR. We also describe the impact on isolation room demand and the time to FebriDx and RT-PCR results.

\section{METHODS}

\section{Patient cohort}

We used data prospectively entered into a COVID-19 triage database and retrospective extraction of clinical and bed allocation data from electronic patient records and hospital IT systems at Northwick Park Hospital, a large district general hospital serving a diverse population in North West London. Patients were included if they required admission to a medical ward from the ED between 10 August 2020 and 4 November 2020 inclusive and had a valid SARS-CoV-2 RT-PCR result on admission.

\section{Triage algorithm}

On initial assessment in the ED, consecutive medical admissions were categorised by the attending clinician into three categories for their likelihood of COVID-19 (unlikely, possible and likely), using clinical criteria such as clinical history, observations and plain chest radiograph based on Public Health England guidance (table 1 and online supplemental table 1). ${ }^{22}$ Patients discharged home or admitted under specialties other than medicine and those under 16 years of age were not triaged using

Table 1 Clinical criteria for determining triage groups, testing strategy and bed allocation from the emergency department (ED) prior to RT-PCR result

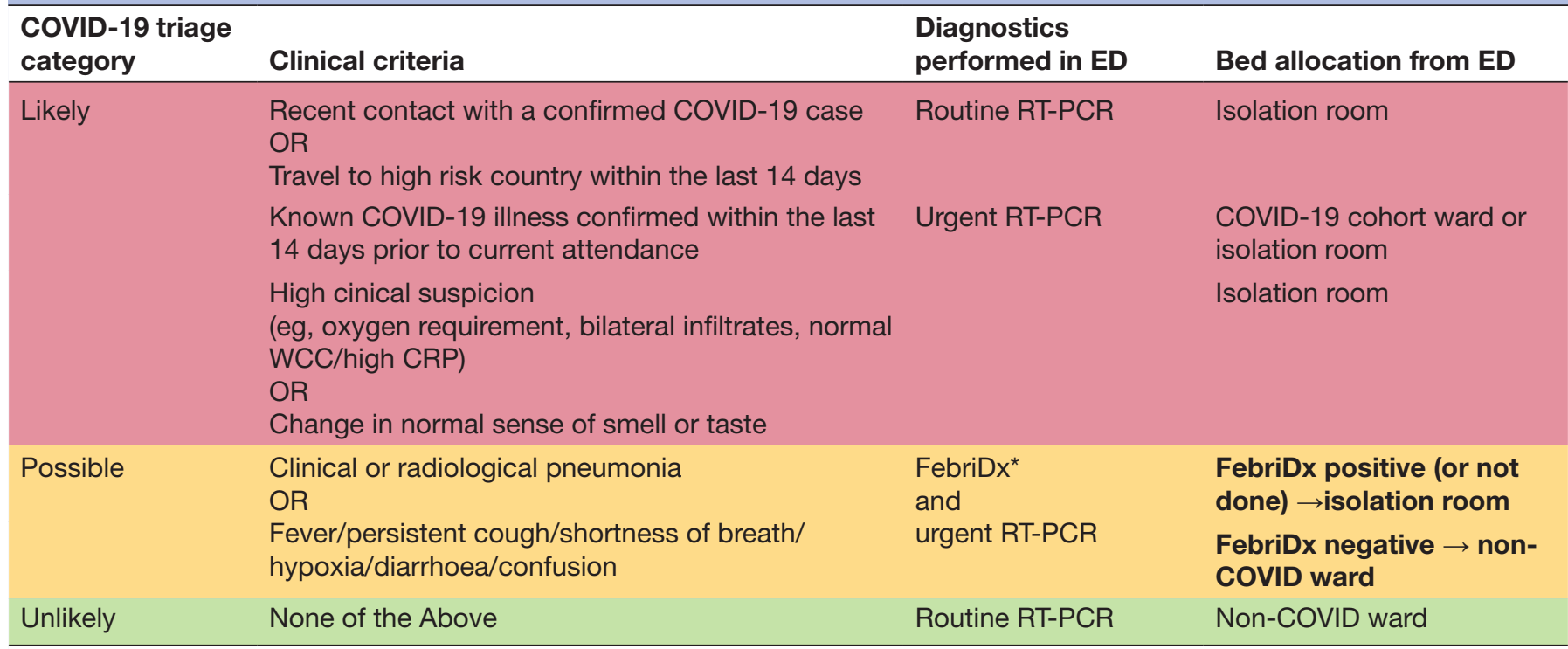

Clinical criteria for determining triage groups are shown as of 8 October 2020. Changes to these criteria over time are detailed in online supplemental table 1.

*Patients were excluded from FebriDx testing if they had a history of COVID-19, were immunosuppressed, required intensive care or high dependency unit admission or had COVID-19 symptoms for $>10$ days.

CRP, C reactive protein; RT-PCR, reverse transcription-PCR; WCC, white cell count. 
the algorithm and did not receive FebriDx testing, therefore their exclusion is unlikely to be a source of ascertainment bias. Patients with epidemiological risk factors for COVID-19 (eg, recent contact with a COVID-19 case or travel to a high-risk country) were triaged as likely COVID-19. We refer to this stage of the triage algorithm as 'clinical criteria'.

We evaluated the impact of using FebriDx in a test-todeisolate strategy among patients designated as possibly having COVID-19 after clinical criteria had been applied at initial assessment. Patients in the possible group underwent testing with FebriDx unless they declined or met an exclusion criterion. Patients were excluded from FebriDx testing if they were immunosuppressed or had symptoms of COVID-19 for $>10$ days (in these situations a measurable type I or type III interferon response might not be present in infected individuals, as per manufacturer's guidance). Patients were also excluded if they had a previous diagnosis of COVID-19 (self-reported or confirmed) or required high dependency unit or intensive care unit admission due to the greater infection control consequences of incorrect triage. All patients underwent NPS testing with SARS-CoV-2 RT-PCR, with rapid RT-PCR assays being prioritised for patients in the likely group.

Only patients with confirmed COVID-19 on SARS-CoV-2 RT-PCR were admitted to a COVID-19 cohort area ('COVID-19 ward'). Those triaged as likely, and those triaged as possible with a positive FebriDx or excluded from having a FebriDx test were designated 'triage positive' and admitted to an isolation room until PCR results were available. Patients assigned to the unlikely COVID-19 group and those with a negative FebriDx test were designated 'triage negative' and admitted to 'nonCOVID wards' while awaiting SARS-CoV-2 RT-PCR results (figure 1 and table 1).

\section{Ethics approval}

FebriDx testing was implemented as part of routine clinical care in response to data on assay performance for COVID-19 and an urgent clinical need. ${ }^{21}$ Results are reported in compliance with Standards for Reporting of Diagnostic Accuracy Studies (STARD) and Strengthening the Reporting of Observational Studies in Epidemiology (STROBE) guidelines (see online supplemental materials). The FebriDx tests were purchased independently

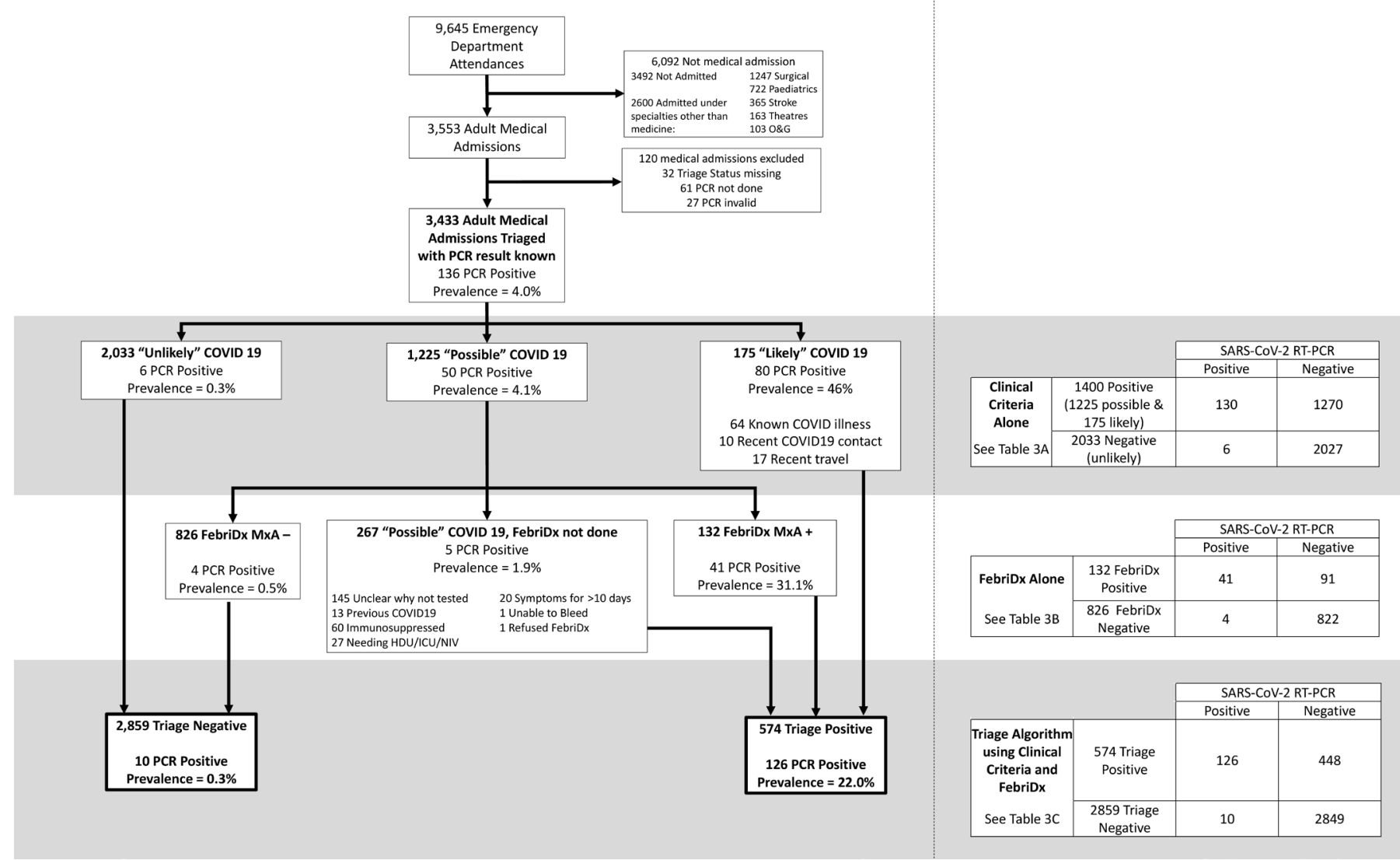

Figure 1 Patient flow through the study and the COVID-19 triage algorithm. Patients were included if they required admission to a medical ward from the emergency department between 10 August 2020 and 4 November 2020 inclusive. Patients were excluded if they were under 16 years of age, admitted under specialties other than medicine or if their triage status or SARSCoV-2 RT-PCR result was unknown. Counts at each stage of triage are shown in $2 \times 2$ tables on the right. These counts correspond with the $2 \times 2$ tables and measures of diagnostic performance shown in table 3 . PCR=SARS-CoV-2 RT-PCR. HDU, high dependency unit; ICU, intensive care unit; NIV, non-invasive ventilation. 
from a UK distributer, and the manufacturer had no role in the study conception, design, data analysis or manuscript preparation.

\section{Testing procedures and definitions}

The FebriDx assay was performed as per the manufacturer's instructions at the point-of-care by trained ED healthcare assistants. In brief, $5 \mu \mathrm{L}$ of capillary blood is placed on the sample window and reagents are released by pressing a button. The result is read after $10 \mathrm{~min}$, with a positive result being the presence of a blue line in the control window and a red line in the $\mathrm{MxA}$ window (limit of detection $40 \mathrm{ng} / \mathrm{mL}$ ). The results from the CRP window were not used given all patients had laboratory CRP measurements. Staff performing FebriDx had access to clinical information but not SARS-CoV-2 RT-PCR results at the time of FebriDx testing. Routine SARS-CoV-2 RT-PCR was done on NPS using either the Panther Fusion SARS-CoV-2 (Hologic, California, USA), Abbott RealTime SARS-CoV-2 (Abbott Park, Illinois, USA) or an extraction-free SARS-CoV-2 RT-PCR assay developed by Health Services Laboratories, UK. ${ }^{23}$ Rapid RT-PCR assays used were Xpert Xpress SARS-CoV-2 (Cepheid, California, USA) or SAMBA II SARS-CoV-2 (Diagnostics for the Real World, California, USA).

Patients were defined as having COVID-19 or not based on the first valid RT-PCR result up to 72 hours after admission. Patients without a valid RT-PCR result or triage status were excluded from the analysis. Vital signs, including National Early Warning Score (NEWS) were recorded on arrival to the ED. All biochemical, haematological and radiological data were from the first results within 48 hours of admission. Thoracic imaging (chest radiographs and CT) were reported and coded based on guidelines on COVID-19 from the British Society of Thoracic Imaging at the time of reporting by radiologists. ${ }^{24}$ Vital status is reported at the time of hospital discharge or data extraction (20 November 2020) for those who were still inpatients.

\section{Data analysis and statistical methods}

We evaluated the real-world diagnostic performance (sensitivity, specificity and positive and negative predictive values with $95 \%$ CIs) of the triage algorithm (described above and in figure 1) using both clinical criteria (described above and in table 1) and the FebriDx assay in combination compared with SARS-CoV-2 RT-PCR from a single NPS as a reference standard. We also evaluated each stage of the triage algorithm independently, estimating measures of diagnostic performance for triage using clinical criteria alone and the FebriDx assay in patients with possible COVID-19 compared with a SARS-CoV-2 RT-PCR reference standard. Patients with missing RT-PCR or those missing data on triaging were excluded from analysis. We also reported the time from arrival to FebriDx and RT-PCR results. We described the proportion of patients with COVID-19 who were correctly isolated, estimated the number of isolation beds made available by FebriDx testing and described the patients with COVID-19 who were incorrectly triaged by the algorithm. Basic descriptive statistics were performed, with comparisons made using $\chi^{2}$ tests for proportions, t-tests for means and Wilcoxon rank sum test to compare nonnormally distributed populations. Logistic regression was used to compare age-adjusted and sex-adjusted estimates of in-hospital death in each triage group, using complete cases only. Statistical analyses were performed using Stata V.14.0 (StataCorp, College Station, Texas, USA). Based on an anticipated sensitivity of $93 \%$, a sample size of 3335 would estimate the sensitivity of the triage algorithm $\pm 5 \%$ with alpha 0.05 and prevalence of $3 \%$.

\section{Patient and public involvement}

There was no patient involvement in the development of the research question, study design or conduct of the study.

\section{RESULTS}

\section{Baseline characteristics and COVID-19 diagnosis}

Between 10 August and 4 November 2020, there were $9645 \mathrm{ED}$ visits resulting in further hospital care. Of these, $3433(35.6 \%)$ were adult medical patients admitted for further treatment, were triaged using the algorithm based on COVID-19 status and had a valid SARS-CoV-2 RT-PCR result (figure 1). One hundred seventy-five (5.1\%) patients were triaged as likely COVID-19, 2033 (59.2\%) patients as unlikely COVID-19 and 1225 (35.7\%) patients were triaged into the possible COVID-19 category. Key patient characteristics are given in table 2 .

There were several differences between the three triage groups (table 2). The likely COVID-19 group were younger, had higher NEWS scores on arrival and more frequently required supplemental oxygen compared with the unlikely group and the possible group $(\mathrm{p}<0.02$ for all comparisons). As expected, more patients in the likely COVID-19 group had chest radiograph changes typical for COVID-19 than in the possible $(p<0.001)$, and the unlikely COVID-19 group $(\mathrm{p}<0.001)$. The possible COVID-19 group were older than the other two groups and were more likely to have an elevated neutrophil count than the likely or possible groups.

Overall, 136/3443 admissions (4.0\%) were diagnosed with PCR-confirmed COVID-19. Prevalence of COVID-19 was $46 \%(80 / 175)$ in likely patients, and $4.1 \%(50 / 1225)$ in the possible group. Of those triaged as unlikely COVID-19, only 6/2033 (0.3\%) were SARS-CoV-2 RT-PCR positive.

\section{Performance of FebriDx and triage algorithm}

The overall diagnostic performance of the clinical triage algorithm compared with the reference standard of SARS-CoV-2 RT-PCR is summarised in table 3. Nine hundred fifty-eight $(78.2 \%)$ patients in the possible group were tested using FebriDx (those excluded are detailed in figure 1); 13.8\% (132/958) of FebriDx test 
Table 2 Baseline characteristics, vital signs, initial investigations, mortality and SARS-CoV-2 RT-PCR results for patients in the unlikely, possible and likely COVID-19 groups

\begin{tabular}{|c|c|c|c|}
\hline Variable & Unlikely & Possible & Likely \\
\hline Age (years) median (IQR) & $69(49,82)$ & $75(60,84)$ & $62(48,74)$ \\
\hline Female sex, n (\%, 95\% Cl) & $969(47.7 \%, 45.5$ to 49.8$)$ & $603(49.2 \%, 46.4$ to 52.0$)$ & $72(41 \%, 34$ to 48$)$ \\
\hline Male sex, $n(\%, 95 \% \mathrm{Cl})$ & $1064(52.3 \%, 50.2$ to 54.5$)$ & $622(50.8 \%, 48.0$ to 53.6$)$ & $103(59 \%, 52$ to 66$)$ \\
\hline Respiratory rate (breaths/min), median (IQR) & $18(18,20)$ & $24(20,28)$ & $24(21,32)$ \\
\hline SpO2 <94\%, n (\%, 95\% Cl) & $61(3.1 \%, 2.4$ to 3.9$)$ & $234(19.5 \%, 17.3$ to 21.8$)$ & $38(22 \%, 17$ to 29$)$ \\
\hline Required supplemental oxygen, $\mathrm{n}(\%, 95 \% \mathrm{Cl})$ & $52(2.7 \%, 2.0$ to 3.4$)$ & $245(20.4 \%, 18.1$ to 22.7$)$ & $52(30 \%, 24$ to 38$)$ \\
\hline Temperature $>37.5^{\circ} \mathrm{C}, \mathrm{n}(\%, 95 \% \mathrm{Cl})$ & $172(8.8 \%, 7.6$ to 10.1$)$ & $359(30.0 \%, 27.4$ to 32.6$)$ & $73(43 \%, 35$ to 50$)$ \\
\hline Chest radiograph - normal, n (\%, 95\% Cl) & $1171(81.0 \%, 79.0$ to 83.0$)$ & $537(49.9 \%, 46.9$ to 52.9$)$ & $42(30 \%, 22$ to 37$)$ \\
\hline Chest CT-typical for COVID-19, n (\%, 95\% Cl) & $0(0 \%, 0$ to 0$)$ & $3(5 \%, 2$ to 16$)$ & $3(43 \%, 10$ to 83$)$ \\
\hline Chest CT-other, n (\%, 95\% Cl) & $26(76 \%, 59$ to 88$)$ & $43(78 \%, 65$ to 87$)$ & $4(57 \%, 17$ to 90$)$ \\
\hline CRP (mg/L), median (IQR) & $5.7(1.4,26.9)$ & $26.4(7.05,87.65)$ & $53.7(25.9,122.7)$ \\
\hline CRP > 20 mg/L, n (\%, 95\% Cl) & $545(28.7 \%, 26.7$ to 30.7$)$ & $656(55.8 \%, 52.9$ to 58.6$)$ & $134(80 \%, 74$ to 86$)$ \\
\hline Lymphocyte count $<1.0 \times 10^{9} / \mathrm{L}, \mathrm{n}(\%, 95 \% \mathrm{Cl})$ & $373(25.3 \%, 23.1$ to 27.5$)$ & $383(43.9 \%, 40.6$ to 47.2$)$ & $70(55 \%, 46$ to 63$)$ \\
\hline Neutrophil count $>7.5 \times 10^{9} / \mathrm{L}, \mathrm{n}(\%, 95 \% \mathrm{Cl})$ & $620(32.0 \%, 29.9$ to 34.0$)$ & $598(50.4 \%, 47.6$ to 53.3$)$ & $61(36 \%, 29$ to 43$)$ \\
\hline Crude in hospital mortality, $\mathrm{n}(\%, 95 \% \mathrm{Cl})$ & $57(2.8 \%, 2.1$ to 3.6$)$ & $89(7.5 \%, 6.0$ to 9.0$)$ & $13(8 \%, 4$ to 12$)$ \\
\hline SARS-CoV-2 RNA detectable on RT-PCR, n (\%, 95\% Cl) & $6(0.3 \%, 0.1$ to 0.5$)$ & $50(4.1 \%, 3.0$ to 5.2$)$ & $80(46 \%, 38$ to 53$)$ \\
\hline
\end{tabular}

For observations on arrival, $3.2 \%-4.1 \%$ of data were missing. Data were missing for $5.5 \%$ of CRP results and $4.0 \%$ of haematology results, $22.4 \%$ of chest radiograph reports and $2.1 \%$ of vital status. Ninety-six patients $(2.8 \%)$ had a chest CT report available. Imaging reports were coded as per BSTI guidelines. Chest radiograph reports were coded as: CVCXO=normal; CVCX1=typical; CVCX2=indeterminate; CVCX3=non-COVID-19. Chest CT reports were coded as: CVCT0=normal; CVCT1=typical; CVCT2=indeterminate; CVCT3=non-COVID-19.

BSTI, British Society of Thoracic Imaging; CRP, C reactive protein; NEWS, National Early Warning Score; SpO2, oxygen saturations.

results were positive for MxA, with $86.2 \%$ negative and no invalid results. The median duration of COVID-19 symptoms in patients tested by FebriDx was 2 days (IQR $1-3, n=847$ ). Patients with positive FebriDx results were younger, more likely to be febrile and less likely to have raised neutrophil counts than FebriDx-negative patients (online supplemental table 2).

Thirty-one per cent $(41 / 132)$ of patients with a positive FebriDx had a positive SARS-CoV-2 RT-PCR, while only 4/826 $(0.5 \%)$ with a negative FebriDx were diagnosed as having COVID-19. All four patients with false-negative FebriDx results had normal chest radiographs. Two patients tested negative for COVID-19 by SARS-CoV-2 RT-PCR but had positive FebriDx results and chest radiograph appearances typical for COVID-19. In the possible COVID-19 group, FebriDx results were available a median of 2.2 hours (IQR 1.4-3.1, n=808) and RT-PCR results a median of 17.8 hours (IQR 11.4-25.3, n=456) after arrival to the ED (figure 2). Eighty-eight per cent of FebriDx results were available within 4 hours of arrival $(n=808)$.

The triage algorithm correctly identified 126/136 patients with PCR-confirmed COVID-19 in the likely group (sensitivity 93\%, 95\% CI 87 to 96) (table 3 ). The 10 patients who were SARS-CoV-2 RT-PCR positive but missed by the triage algorithm are described in online supplemental table 3. Six out of ten patients were classified as unlikely, and 4/10 were classified as possible COVID-19 and had a negative FebriDx; 2/10 were febrile on admission, none required supplemental oxygen, length of stay was short (median 2 days) and 8 had normal chest radiographs (2 did not have thoracic imaging done). Specificity of the algorithm was $86.4 \%$ (95\% CI 85.2 to 87.5 ), and negative predictive value was $99.7 \%$ (95\% CI 99.4 to 99.8 ). Although changes were made to clinical triage criteria during the study period (online supplemental table 1), our estimates of diagnostic performance were comparable after excluding individuals who arrived before the last alteration (online supplemental table 4).

\section{Outcomes}

Ninety-five per cent (129/136) of patients with COVID-19 were appropriately managed in isolation rooms or COVID-19 cohort wards as a result of the triage algorithm (online supplemental table 5). Of the 10 patients with PCR-confirmed COVID-19 not identified by the triage algorithm, 7 were initially managed in a non-COVID ward. Had all patients been isolated until SARS-CoV-2 RT-PCR result was available (ie, without using clinical criteria or 
Table 3 Cross tabulation of results of the triage algorithm with and without FebriDx as well as the results of FebriDx within the possible COVID-19 group compared with a SARS-CoV-2 RT-PCR reference standard

\begin{tabular}{|c|c|c|c|c|c|}
\hline \multirow[b]{2}{*}{ A } & & \multicolumn{3}{|c|}{ SARS-CoV-2 RT-PCR } & \multirow{5}{*}{$\begin{array}{l}\text { PPV: } \\
9.3 \% \\
\text { (95\% Cl } 7.9 \text { to } 10.9 \text { ) } \\
\text { NPV: } \\
99.7 \% \\
(95 \% \mathrm{Cl} 99.3 \text { to } 99.9)\end{array}$} \\
\hline & & Positive & Negative & Total & \\
\hline \multirow[t]{6}{*}{$\begin{array}{l}\text { Clinical criteria } \\
\text { alone (without } \\
\text { FebriDx) } \\
(n=3433)\end{array}$} & $\begin{array}{l}\text { Likely or } \\
\text { possible } \\
\text { COVID-19 }\end{array}$ & 130 & 1270 & 1400 & \\
\hline & $\begin{array}{l}\text { Unlikely } \\
\text { COVID-19 }\end{array}$ & 6 & 2027 & 2033 & \\
\hline & & & & & \\
\hline & Total & 136 & 3297 & 3433 & \\
\hline & & Sensitivity & Specificity & & \\
\hline & & $\begin{array}{l}96 \% \\
(95 \% \text { Cl } 91 \text { to } 98)\end{array}$ & $\begin{array}{l}61.5 \% \\
(95 \% \text { Cl59.8 to } 63.1)\end{array}$ & & \\
\hline
\end{tabular}

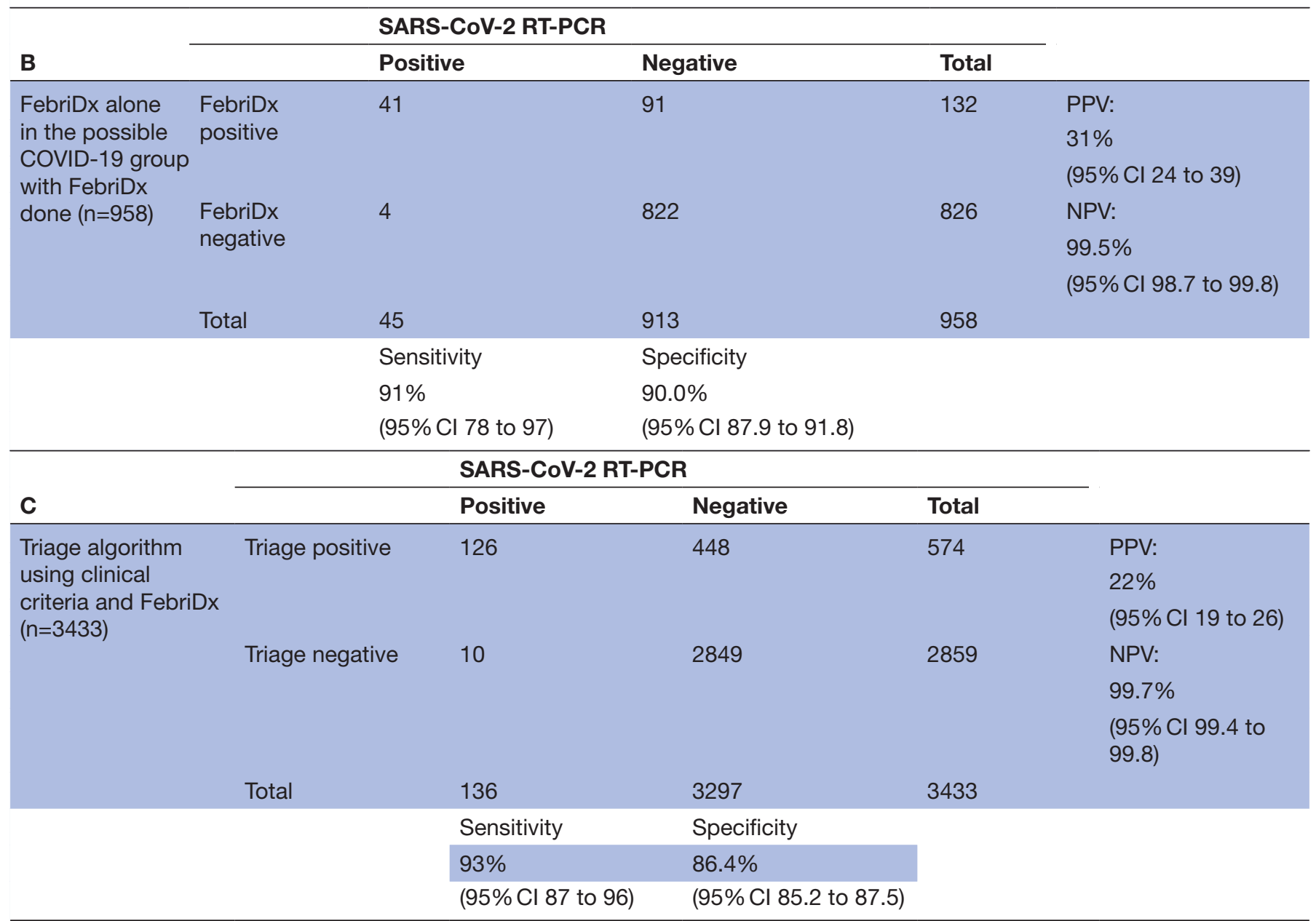

Measures of diagnostic performance are presented for the triage algorithm for the detection of COVID-19: (3A) using clinical criteria alone without FebriDx, where subjects are classified as positive or negative based on clinical criteria shown in table 1. Subjects were 'positive' if they were assigned as likely or possible COVID-19 based on clinical criteria alone. (3B) Using the FebriDx assay alone within the possible COVID-19 group receiving a FebriDx test. Subjects are classed as FebriDx positive or negative based on the FebriDx test only. (3C) Using clinical criteria supported by the FebriDx assay. Subjects were classed as triage positive or negative based on their flow through the algorithm as shown in figure 1. Patients were triage positive if they were triaged as likely COVID-19 or possible COVID-19 without a negative FebriDx result. Patients were triage negative if they were triaged as unlikely COVID-19 or possible COVID-19 with a negative FebriDx result. NPV, negative predictive value; PPV, positive predictive value. 


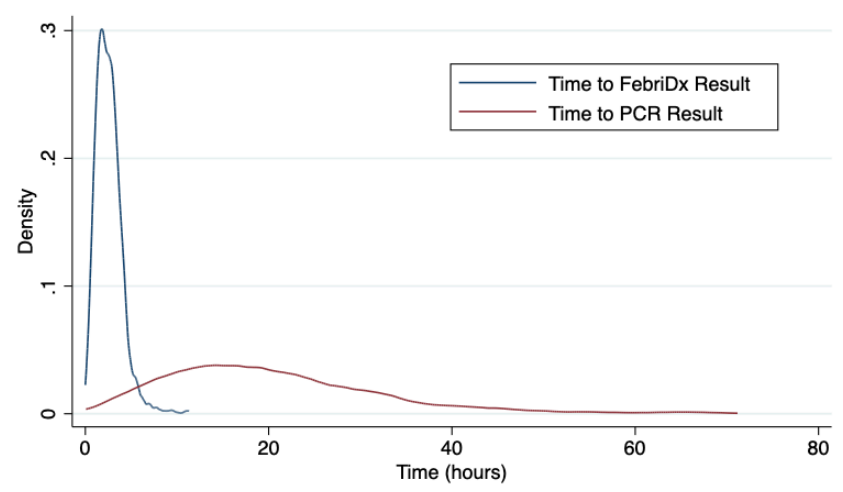

Figure 2 Time from arrival to the availability of FebriDx and SARS-CoV-2 RT-PCR results. Kernel frequency density plot using the Epanechnikov function; time to FebriDx result was calculated as the time from arrival to the emergency department until the time the FebriDx result was recorded (blue plot), bandwidth=0.3; time to RT-PCR result was calculated as the time from arrival to the emergency department until the time the SARS-CoV-2 RT-PCR result was recorded (red plot), bandwidth $=2$.

FebriDx to de-isolate), 2859 more isolation rooms would have been used. When using the triage algorithm, clinical criteria allowed 2033 patients to be released from isolation after being classified as unlikely COVID-19. The addition of FebriDx to clinical triage allowed 826 more patients to be managed in 'non-COVID' wards than if all patients triaged possible COVID-19 had required isolation (9.5 isolation rooms saved per day, $95 \%$ CI 8.9 to 10.2 ).

Eleven (8\%) patients with COVID-19 died compared with $150 \quad(4.5 \%)$ without COVID-19 $(\mathrm{p}=0.042)$. Ageadjusted and sex-adjusted odds of death during the admission were higher for patients in the likely (OR $3.42,95 \%$ CI 1.81 to 6.45 ) and possible groups (OR 2.44, $95 \%$ CI 1.73 to 3.44 ) than the unlikely COVID-19 group.

\section{DISCUSSION}

Our main findings are that a pragmatic triage algorithm using simple clinical parameters available within the ED and the FebriDx point-of-care test had good sensitivity (93\%) and excellent NPV (99.7\%) for COVID-19 diagnosed by RT-PCR. Inclusion of FebriDx improved the specificity of triage with minimal reductions in sensitivity, allowing a substantial reduction in the number of isolation rooms needed.

Although clinicians were able to identify patients likely and unlikely to have COVID-19 (46\% and $0.3 \%$ of whom had confirmed COVID-19, respectively) based on clinical assessment, radiology and basic blood tests, their assessment was not sufficiently specific. The group of patients identified as 'possible' COVID-19 had a 4\% prevalence of COVID-19, high mortality and was large enough to overwhelm isolation room capacity. We demonstrate a simple, rapid test performed at the point-of-care can help further risk stratify this group. In real-life settings in a busy $\mathrm{ED}$, a point-of-care test was able to inform isolation decisions within 4 hours of arrival compared with PCR results which were too slow to inform patient flow from ED, even when using 'rapid' PCR assays. Although formal cost-effectiveness analysis was not performed, each FebriDx test only costs about US $\$ 18$, and this may lead to cost savings.

The strengths of this study are its pragmatic design under routine clinical settings, and that we are able to account for over $95 \%$ of medical admissions, reducing risks of bias. There are, however, several limitations. A single SARS-CoV-2 RT-PCR is an imperfect reference standard, and does not account for RT-PCR-negative patients with COVID-19. We used multiple RT-PCR platforms, which will have different PCR targets and performance. Ten per cent of patients in the possible group did not get tested with FebriDx for unclear reasons, which could be a source of bias unless these were unavoidable random losses in a busy ED. The prevalence of COVID-19 was $4.0 \%$ in this cohort, and it is unclear what impact a higher prevalence of COVID-19 or other respiratory pathogens such as influenza would have on these findings. The criteria for likely and possible COVID-19 groups changed during the study period, although this is unlikely to significantly alter the outcomes.

These data build on previous studies of FebriDx showing good sensitivity, and utility as a 'rule-out' test for COVID$19 .{ }^{17-20}$ In our pragmatic, 'real-world' study, clinical triage by ED clinicians was imperfect. For example, two PCR-positive patients were incorrectly classified as 'unlikely' COVID-19 given they had a temperature of $>38^{\circ} \mathrm{C}$ on arrival (online supplemental table 3). The estimate of sensitivity of FebriDx for detecting COVID-19 in our cohort is lower than previously described, likely because our testing strategy differs in that it does not include patients deemed likely to have COVID-19 by clinical criteria. Testing this group would have been unlikely to alter clinical decisions, even if FebriDx had been negative, given the high pretest probability. The FebriDx test allowed patients with possible COVID-19 to be divided into two groups with similar characteristics and clinical features, but vastly different COVID-19 prevalence $(0.5 \%$ in FebriDx negative, and $31 \%$ in FebriDx positive). However, about $10 \%$ of patients in this group were not eligible for FebriDx testing, and had to be managed in isolation rooms as triage-positive patients (see figure 1).

Only 10 patients with COVID-19 were incorrectly triaged by the algorithm, 4 of whom were tested and 'missed' using FebriDx. These patients were younger, less symptomatic, did not have chest radiograph changes and mostly likely had mild or asymptomatic COVID-19 infection. Given that MxA is an intracellular GTPase induced by type I and type III interferon responses, it is plausible that sensitivity would be lower in oligosymptomatic or asymptomatic infection. ${ }^{25}$ Although the patients missed by the algorithm are potential sources of nosocomial transmission, asymptomatic disease is thought to be less transmissible. ${ }^{26}$ We found no nosocomial cases related to these patients.

In conclusion, we demonstrate that a simple triage system including the novel FebriDx point-of-care test had good 
sensitivity and negative predictive value for COVID-19 and utility for managing medical admissions from the ED.

\section{Author affiliations}

${ }^{1}$ Department of Infectious Diseases, Northwick Park Hospital, London North West University Healthcare NHS Trust, Harrow, UK

${ }^{2}$ Department of Acute Medicine, Northwick Park Hospital, London North West University Healthcare NHS Trust, Harrow, UK

${ }^{3}$ School of Medicine, Imperial College London, London, UK

${ }^{4}$ Transformation Programme Director, London North West University Healthcare NHS Trust, London, UK

${ }^{5}$ Emergency Department, Northwick Park Hospital, London North West University Healthcare NHS Trust, Harrow, UK

${ }^{6}$ Department of Pathology, Northwick Park Hospital, London North West University Healthcare NHS Trust, Harrow, UK

${ }^{7}$ Department of Microbiology, Northwick Park Hospital, London North West University Healthcare NHS Trust, Harrow, UK

${ }^{8}$ Department of Medicine, Imperial College London, London, UK

${ }^{9}$ Institute for Global Health, University College London, London, UK

${ }^{10}$ Clinical Research Department, London School of Hygiene \& Tropical Medicine, London, UK

\section{Twitter Hamish Houston @HamishHouston2 and Ankur Gupta-Wright @ankurgw}

Acknowledgements We would like to acknowledge all the clinical staff at Northwick Park Hospital who cared for the patients involved in this study. In particular, we would like to thank the point-of-care team for their outstanding work in establishing and running the new point-of-care testing service in the emergency department.

Contributors HH, AG-W, LJ, SAF, JB-L, JR, NV, NV, GGR and AKA made substantial contribution to the conception of the work. HH, AG-W, LJ and GD made substantial contribution to the design of the work. HH, GD, SN, KS, SP, MGD and MT contributed to data acquisition. HH and AGW analysed the data. HH, AGW and LJ contributed to data interpretation. HH and AGW drafted the manuscript. All authors contributed to revising the manuscript critically for important intellectual content, approved the final manuscript and are accountable for all aspects of the work.

Funding The authors have not declared a specific grant for this research from any funding agency in the public, commercial or not-for-profit sectors.

Competing interests None declared.

Patient consent for publication Not required.

Ethics approval The study was approved by the London North West University Hospitals Trust Research and Development Committee (SE20/069), and given this was a retrospective review using routinely collected clinical data, they deemed formal ethical approval was not required.

Provenance and peer review Not commissioned; externally peer reviewed.

Data availability statement Data are available on reasonable request. Data are available on reasonable request, subject to approval by the London North West University Healthcare NHS Trust Research and Governance Department and approval from relevant ethics and regulatory bodies.

Supplemental material This content has been supplied by the author(s). It has not been vetted by BMJ Publishing Group Limited (BMJ) and may not have been peer-reviewed. Any opinions or recommendations discussed are solely those of the author(s) and are not endorsed by BMJ. BMJ disclaims all liability and responsibility arising from any reliance placed on the content. Where the content includes any translated material, BMJ does not warrant the accuracy and reliability of the translations (including but not limited to local regulations, clinical guidelines, terminology, drug names and drug dosages), and is not responsible for any error and/or omissions arising from translation and adaptation or otherwise.

Open access This is an open access article distributed in accordance with the Creative Commons Attribution Non Commercial (CC BY-NC 4.0) license, which permits others to distribute, remix, adapt, build upon this work non-commercially, and license their derivative works on different terms, provided the original work is properly cited, appropriate credit is given, any changes made indicated, and the use is non-commercial. See: http://creativecommons.org/licenses/by-nc/4.0/.

\section{ORCID iDs}

Hamish Houston http://orcid.org/0000-0001-5302-7563
Kamal Shah http://orcid.org/0000-0003-2341-7688

Guduru Gopal Rao http://orcid.org/0000-0002-8108-4431

Ankur Gupta-Wright http://orcid.org/0000-0002-5150-2970

\section{REFERENCES}

1 Verelst F, Kuylen E, Beutels P. Indications for healthcare surge capacity in European countries facing an exponential increase in coronavirus disease (COVID-19) cases, March 2020. Euro Surveill 2020;25:2000323.

2 Hui KPY, Cheung M-C, Perera RAPM, et al. Tropism, replication competence, and innate immune responses of the coronavirus SARS-CoV-2 in human respiratory tract and conjunctiva: an analysis in ex-vivo and in-vitro cultures. Lancet Respir Med 2020;8:687-95.

3 Zhou J, Otter JA, Price JR, et al. Investigating SARS-CoV-2 surface and air contamination in an acute healthcare setting during the peak of the COVID-19 pandemic in London. Clin Infect Dis 2020:ciaa905.

4 van Doremalen N, Bushmaker T, Morris DH, et al. Aerosol and surface stability of SARS-CoV-2 as compared with SARS-CoV-1. N Engl J Med 2020;382:1564-7.

5 Cevik M, Marcus JL, Buckee C. Severe acute respiratory syndrome coronavirus 2 (SARS-CoV-2) transmission dynamics should inform policy. Clin Infect Dis 2020;41:ciaa1442.

6 Rickman HM, Rampling T, Shaw K, et al. Nosocomial transmission of coronavirus disease 2019: a retrospective study of 66 hospitalacquired cases in a London teaching hospital. Clin Infect Dis 2021;72:690-3.

7 Wang D, Hu B, Hu C, et al. Clinical characteristics of 138 hospitalized patients with 2019 novel coronavirus-infected pneumonia in Wuhan, China. JAMA 2020;323:1061-9.

8 Brendish NJ, Poole S, Naidu VV, et al. Clinical impact of molecular point-of-care testing for suspected COVID-19 in hospital (COV19POC): a prospective, interventional, non-randomised, controlled study. Lancet Respir Med 2020;8:1192-200.

9 NHS England. Pillar 1 NHS Labs COVID-19 testing turnaround time data: 15 July 2020, 2020. Available: https://www.england.nhs.uk/ coronavirus/publication/nhs-labs-covid-19-testing-turnaround-timedata/

10 Gibani MM, Toumazou C, Sohbati M, et al. Assessing a novel, labfree, point-of-care test for SARS-CoV-2 (CovidNudge): a diagnostic accuracy study. Lancet Microbe 2020;1:e300-7.

11 Collier DA, Assennato SM, Warne B, et al. Point of care nucleic acid testing for SARS-CoV-2 in hospitalized patients: a clinical validation trial and implementation study. Cell Rep Med 2020;1:100062.

12 Fink DL, Khan PY, Goldman N, et al. Development and internal validation of a diagnostic prediction model for COVID-19 at time of admission to hospital. QJM 2020;5:hcaa305.

13 Wynants L, Van Calster B, Collins GS, et al. Prediction models for diagnosis and prognosis of covid-19: systematic review and critical appraisal. BMJ 2020;369:m1328.

14 Engelmann I, Dubos F, Lobert P-E, et al. Diagnosis of viral infections using myxovirus resistance protein $\mathrm{A}(\mathrm{M} \times \mathrm{A})$. Pediatrics 2015;135:e985-93.

15 Nakabayashi M, Adachi Y, Itazawa T, et al. MxA-based recognition of viral illness in febrile children by a whole blood assay. Pediatr Res 2006;60:770-4.

16 Sambursky R, Shapiro N. Evaluation of a combined MxA and CRP point-of-care immunoassay to identify viral and/or bacterial immune response in patients with acute febrile respiratory infection. Eur Clin Respir J 2015;2:28245.

17 Thomas J, Pociute A, Kevalas R, et al. Blood biomarkers differentiating viral versus bacterial pneumonia aetiology: a literature review. Ital J Pediatr 2020;46:4.

18 Clark TW, Brendish NJ, Poole S, et al. Diagnostic accuracy of the FebriDx host response point-of-care test in patients hospitalised with suspected COVID-19. J Infect 2020;81:607-13.

19 Karim N, Ashraf MZ, Naeem M, et al. Utility of the FebriDx point-ofcare test for rapid triage and identification of possible coronavirus disease 2019 (COVID-19). Int J Clin Pract 2021;75:e13702.

20 National Institute for Health and Care Excellence. Overview | FebriDx for $\mathrm{C}$-reactive protein and myxovirus resistance protein A testing | advice | NICE, 2020. Available: https://www.nice.org.uk/advice/ mib224/

21 Ismail SA, Huntley C, Post N, et al. Horses for courses? assessing the potential value of a surrogate, point-of-care test for SARS-CoV-2 epidemic control. Influenza Other Respi Viruses 2021;15:3-6.

22 Public Health England. (2020, June 18) COVID-19: infection prevention and control guidance, 2020. Available: https://www. publichealth.hscni.net/sites/default/files/2020-10/COVID-19_ 
Infection_prevention_and_control_guidance_complete. $3.2 \% 2818$ 06_2020\%29_0.pdf

23 Grant P, Turner M, Shin GY. Extraction-free COVID-19 (SARS-CoV-2) diagnosis by RT-PCR to increase capacity for national testing programmes during a pandemic. bioRxiv 2020.

24 British Society of Thoracic Imaging. BSTI COVID-19 CXR report Proforma, 2020. Available: https://www.google.com/url?sa=t\&rct=j\& $q=\& e s r c=s \&$ source $=$ web\&cd $=\& v e d=2 a h U K E w j j$ j4CurtrtAhUnQUEA HRTWA80QFjABegQIARAC\&url=https\%3A\%2F\%2Fwww.bsti.
org.uk\%2Fmedia\%2Fresources\%2Ffiles\%2FBSTI_COVID_CXR_ Proforma_v.3-1.pdf\&usg=AOvVaw0vUxoui2fz68LEbrs6eTQm

25 Shirley M. FebriDx®: a rapid diagnostic test for differentiating bacterial and viral aetiologies in acute respiratory infections. Mol Diagn Ther 2019;23:803-9.

26 Cevik M, Tate M, Lloyd O, et al. SARS-CoV-2, SARS-CoV, and MERS-CoV viral load dynamics, duration of viral shedding, and infectiousness: a systematic review and meta-analysis. Lancet Microbe 2021;2:e13-e22. 